\title{
Design and Implementation of Fuzzy PID Control for Oil Temperature in Overvoltage Withstand Test
}

\author{
Xiayi Hao, Genghuang Yang \\ School of Automation and Electrical Engineering \\ Tianjin University of Technology and Education \\ Tianjin, China \\ tutezdh1203@163.com
}

\author{
Xin $\mathrm{Su}$, Xiaotian Xu \\ Chengxi Power Supply Branch \\ Tianjin Electrical Power Company \\ Tianjin, China \\ ygenghuang@126.com
}

\begin{abstract}
It is difficult to control the temperature, due to the specific heating of the insulating oil and the high viscosity. It is necessary to design other method to control the temperature rather than the conventional control method. Combined with some characteristics of temperature control, a method of fuzzy PID control is proposed and validated by MATLAB for the adaptive control ability. The simulation results show that the control strategy has good robustness and stability, and the control precision is higher than conventional PID control.
\end{abstract}

Keywords-fuzzy control; control algorithm; temperature control; MATLAB simulation

\section{INTRODUCTION}

The traditional pressure tester does not consider the control of the ambient temperature of the tested sample. In this paper, the temperature control of the insulating oil of the test cup in the pressure tester is studied. The specific practice is to intercept part of the XLPE (cross-linked polyethylene) into the pressure test cup and fix it, and the cup is filled with insulating oil. The temperature of insulating oil is the ambient temperature of XLPE. This paper uses the 25\# transformer oil as insulating oil. Through regulating the temperature of the insulating oil, it can achieve the purpose of controlling the ambient temperature around the XLPE. The heating is carried out mainly by heater strip. The heat generated by heater strip at the time of operation causes the oil around it to heat up and expand. Through the oil up and down convection, the heat spread to the upper cup. The balance of heating is achieved through the combination of heater strip and stirrer. The temperature of the insulating oil is controlled by the algorithm studied in this paper to ensure the stability of the ambient temperature of XLPE.

\section{A. PID control}

PID control is simple, easy to implement, wide application and strong stability, so it is widely used in industrial control. According to statistics, in the field of industrial control, the proportion of PID control and its associated optimization control up to $90 \%$. Even in the rapid development of advanced control technology, PID control technology is still the first to consider the method and occupy the most important position in the application. The performance of PID control mainly

This paper is supported by the project of Tianjin University of Technology and Education (XJKC030155 and (XJKC031112) depends on the tuning of three parameters of P, I and D. Only to set the appropriate parameters, PID controller can achieve optimal control to meet the control speed and accuracy.

\section{B. Theoretical feasibility of PID}

The general block diagram of PID control system is shown in Fig.1.

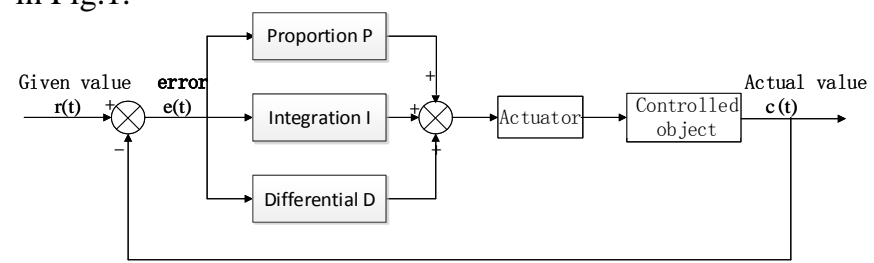

Fig. 1. Schematic diagram of a conventional PID control systemn

From Figure 1, we can deduce the relationship between $r(t)$, $c(t)$ and $e(t)$ in the PID controller

$$
e(t)=r(t)-c(t)
$$

$e(t)$, respectively, through the proportion of links, integral links and differential links, and then the three linear combination, can constitute the control amount $u(t)$, So as to control the controlled object, the continuous form is

$$
u(t)=K_{P}\left[e(t)+\frac{1}{T_{I}} \int_{0}^{t} e(t) d t+T_{D} \frac{d e(t)}{d t}\right]
$$

Where $K_{P}$ is the coeffcient of proportionality; $T_{I}$ is the integral time constant, and $\mathrm{T}_{\mathrm{D}}$ is the derivative time constant

Under normal circumstances, a complete PID controller is composed of the proportion of links, integral links and differential links of the three links, the various links in the control process played by the role is different, the specific control of each link described as follows:

Proportional adjustment function: the real-time proportional to the actual value of the system and the reference value between the deviation $e(t)$ to respond, as long as the control system bias, the system immediately targeted control to reduce the bias. The proportional coefficient $\mathrm{K}_{\mathrm{P}}$ directly 
determines the strength of the proportional adjustment effect. The larger the $K_{P}$, the smaller the steady-state error, but will increase the overshoot accordingly, which will lead to oscillation and make the control system not stable enough.

Integral regulation: This link is mainly used to eliminate static errors. The integral adjustment effect is determined by the value of $T_{I}$. The $T_{I}$ is larger, the weaker the integral effect will be, the corresponding overshoot will decrease, whereas the integral action is strong, also can decrease the static error, but the overshoot will increase, therefore, it should pay attention to the balance between them.

Differential regulation: This part of the transient change of the reaction system deviation, ahead control, can effectively improve the dynamic performance of the system. The magnitude of the differential regulation is determined by the value of the $T_{D}$, and the $T_{D}$ is greater, the stronger the effect of the error suppression, the smaller the $T_{D}$ value, the weaker the effect of the error variation, and the differential regulation to amplify the noise. When the differential control effect is too strong, it will reduce the system's anti-jamming capability.

\section{FUZZY PID CONTROL}

\section{A. Basic principles of fuzzy control}

Fuzzy control is an algorithm based on fuzzy logic. The principle is that the state of the controlled object is blurred into the fuzzy quantity described in the human language in the control process, then, according to the language control rules formulated by the actual control experience, the fuzzy value of the output control quantity is obtained by fuzzy reasoning. Finally, the fuzzy value of the control quantity is converted into the precise control quantity that the actuator can play a role in the control.

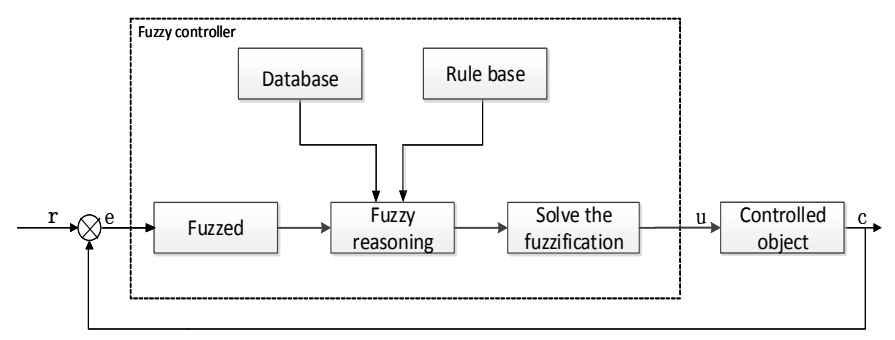

Fig. 2. Fuzzy control system structure

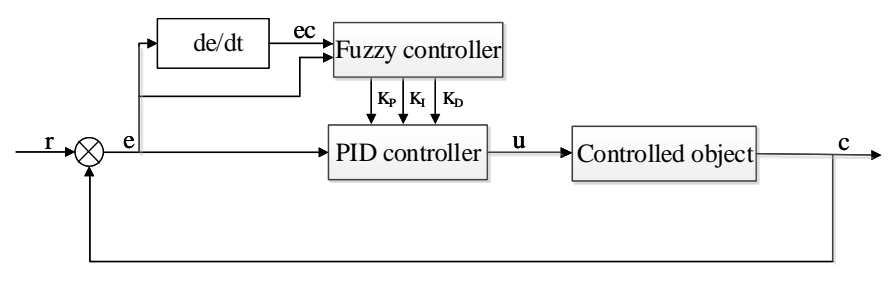

Fig.3. Fuzzy control system structure

As can be seen from the figure, the fuzzy controller consists of the following four parts.

1) Fuzzy module. The function of the fuzzy module is to change the value of the precise input and output related to the control to the fuzzy language value through certain rules. The design step is actually the process of defining language variables on the domain.

2) Knowledge base. Knowledge base is composed of two parts: database and rule base. The database includes the definition of the fuzzy variable and the membership function. The rule base is mainly composed of fuzzy language control rules, the main basis is to sum up the practical experience in the control.

3) Fuzzy reasoning module. Fuzzy reasoning is based on fuzzy input and related data, the appropriate rules derived output fuzzy value.

4) Defuzzification module. The de-fuzzification is to convert the fuzzy output obtained by fuzzy reasoning into the precise control quantity to achieve the precise control of the actuator. At present, the conversion method widely used is the weighted average method and the maximum membership method.

\section{B. Fuzzy self-tuning PID control}

Fuzzy control has two very obvious advantages: first it can rely on people in the production practice of control experience, in which case the control task is not necessary to establish a precise model of the controlled object; Second, the fuzzy control is suitable for the control of the system with hysteresis, because of its stability, fast response, overshoot small. The shortcomings are obvious, it is difficult to summarize the fuzzy control rules.

Once the rule is established, it cannot be changed online, in addition to the fuzzy controller is not integral part, so the steady-state accuracy is not high.

Therefore, the combination of fuzzy control and traditional PID control, the use of fuzzy self-tuning PID parameters of the complementary advantages, so fast and accurate to complete the control task, the specific system structure shown in Fig.3. The fuzzy controller performs fuzzy reasoning based on the input quantities $e$ and $e c$, outputs the precise PID control parameters $K_{P}, K_{I}$ and $K_{D}$, and then controls the greenhouse temperature by the PID controller.

\section{FUZZY SELF-TUNING PID CONTROL ALGORITHM}

In this design, the design steps of the fuzzy module are as follows.

1) Determine input and output variables. When the control quantity is adjusted in the field, it is mainly controlled according to the actual output and output rate of change. Therefore, the error e and the error rate of change ec are taken as the input of the fuzzy module in this system. The fuzzy controller has three output controls, namely " $\triangle K_{P} ", " \triangle K_{I} "$ and " $\triangle K_{D}$ ". In this system, set the temperature $T$, then $\mathrm{e}=\mathrm{T}$ set value-T current value, ec $=d$ (e current value-e on the moment) / $d t$. Combined with the actual situation of field control, the range of positive and negative direction of the error e can not be symmetrical in the system design, otherwise the overshoot will be too large, Therefore, the value of the input value $e$ is in the range of $[-4,12]$, the range of the value of ec is 
[-1,1], the output value $\triangle K_{P}$ is in the range of [-900,900], and the range of $\triangle K_{I}$ is [-12,12], $\triangle K_{D}$ is in the range [-6000,6000].

2) Design language variable domain. In this paper, we define the language variables "error E" and "error change EC" respectively on the domain of e and ec, and define the language variable "control quantity $\triangle K_{P}$ " on the domain of $\triangle K_{P}, \triangle K_{I}$, $\triangle K_{D}$, "Control amount $\triangle K_{I}$ ", "control amount $\triangle K_{D}$ ". In the fuzzy controller, the domain of the language variable is usually a finite discrete integer.

In the fuzzy control system, the quantization factor $\mathrm{Ke}=$ $1 / 2, \mathrm{Kec}=6$, the scale factor $\mathrm{K} \triangle \mathrm{K}_{\mathrm{P}}=150, \mathrm{~K} \triangle \mathrm{K}_{\mathrm{I}}=2, \mathrm{~K} \triangle$ $\mathrm{K}_{\mathrm{D}}=1000$. Fuzzy controller input

$$
\begin{gathered}
E=<k_{e} \cdot\left(e-\frac{e_{H}+e_{L}}{2}\right)>, \\
E C=<k_{e c} \times\left(e c-\frac{e c_{H}+e c_{L}}{2}\right)>.
\end{gathered}
$$

\section{<> Represents rounding.}

3) Define the language value of the variable. The fuzzy subset of $\mathrm{E}$ is negative, $\mathrm{ZO}$ (zero), PS (positive), PM (median), $\mathrm{PB}$ (positive) $\}, \mathrm{EC}$ and $\triangle \mathrm{K}_{\mathrm{P}}, \triangle \mathrm{K}_{\mathrm{I}}, \triangle \mathrm{K}_{\mathrm{D}}$ \{NB (negative), NM (negative), NS (negative), $\mathrm{ZO}$ (zero), PS (positive), $\mathrm{PM}$ (median), PB (positive)\}.

4) Determine membership function. The two inputs of the fuzzy controller, the input range of the error $\mathrm{E}$ is moderate, and the input range of EC is too small. Therefore, both of them are suitable for the triangular membership functions with higher resolution. Since the value of the three outputs is magnified by a factor of 1000, the effect of the actual temperature on the need for rapid sensitivity, so the same use of three-star membership function. Triangle function is characterized by sharp shape, so the resolution is high, the output caused by the output changes are relatively large, with high control sensitivity.

5) Establish fuzzy control rules. PID parameter tuning, which takes into account the entire control process, $K_{P} 、 K_{I}$, $\mathrm{K}_{\mathrm{D}}$ three parameters each play a role in the control process, and the effect of each in control. The key to fuzzy PID self-tuning control is to rely on the practical experience of the relevant control process, through the practice of continuous testing, modification, summary, and thus establish a good control performance and meet the control requirements of the control rules.

The control rules of $\triangle K_{P} . \triangle K_{P}$ value directly related to the controller in the system response speed, can make the system bias smaller. In the early stages of adjustment, $\triangle K_{P}$ should take a large value to speed up the system response. In the middle of the adjustment, $\triangle K_{P}$ should be appropriately reduced, while ensuring the response speed to prevent excessive overshoot. In the late adjustment, $\triangle K_{P}$ value should be moderate or smaller, it is necessary to maintain the system stability should also prevent excessive overshoot.
The control rules of $\triangle K_{I} . \triangle K_{I}$ in the control of the role of the system to reduce the steady-state error. In the preadjustment period, in order to effectively prevent the integral saturation and a large overshoot, therefore, $\triangle K_{I}$ value is smaller, usually take 0 . In the middle of the regulation, in order to play the role of integral, it should be appropriate to increase the value of $\triangle K_{I}$. In the later stage of regulation, the value of $\triangle K_{\mathrm{I}}$ is large, which can effectively reduce the static error of the system.

The control rules of $\triangle K_{D} . \triangle K_{D}$ is mainly used to control the dynamic operating characteristics of the system. In the early stage of adjustment, $\triangle K_{D}$ value is larger, in order to increase the differential effect, thereby reducing the final overshoot. In the middle of the adjustment, $\triangle K_{D}$ changes on the dynamic characteristics of relatively large, so $\triangle K_{D}$ value should be appropriate to reduce. In the later stages of regulation, the value of $\triangle K_{D}$ is small in order to effectively reduce the effect of disturbance.

6) Solve the fuzzification. The fuzzy output value is obtained by querying the fuzzy control rule table, and then the fuzzy control process is transformed into the precise control quantity to realize the control of the actuator. The fuzzy language variable of the output is obtained by querying the rule table, and then the fuzzy control can be obtained by determining the membership degree of the fuzzy language variable. In the process of solving the fuzzy process, the system uses the maximum membership method.

\section{THE EFFECT OF FUZZY PID CONTROL IN TEMPERATURE CONTROL}

In the experiment, two methods of conventional PID control and fuzzy self-tuning PID control are taken respectively, and the final control effect is recorded respectively. Statistics the thermometer to display the data, recorded every 30 seconds, and according to this data to draw out the control effect map.

Fig.4 and Fig.5 show the temperature control effect of the conventional PID and fuzzy self-tuning PID control when the temperature is $30^{\circ} \mathrm{C}$. It is found that fuzzy self-tuning PID control can effectively improve the oversampling problem of conventional PID control. This control method also shortens the time to stabilize, and the final result satisfies the requirement that the error be within $\pm 2{ }^{\circ} \mathrm{C}$.

At the same time as the insulating oil and room temperature difference between the larger heat dissipation is better, cooling time required significantly reduced.

Fig.6 and Fig.7 show the temperature control effect of the conventional PID and fuzzy self-tuning PID control when the temperature is $90^{\circ} \mathrm{C}$. By comparing Fig.6 and Fig.7, it can be seen that fuzzy self-tuning PID control can appropriately reduce overshoot and improve dynamic characteristics over conventional PID. 


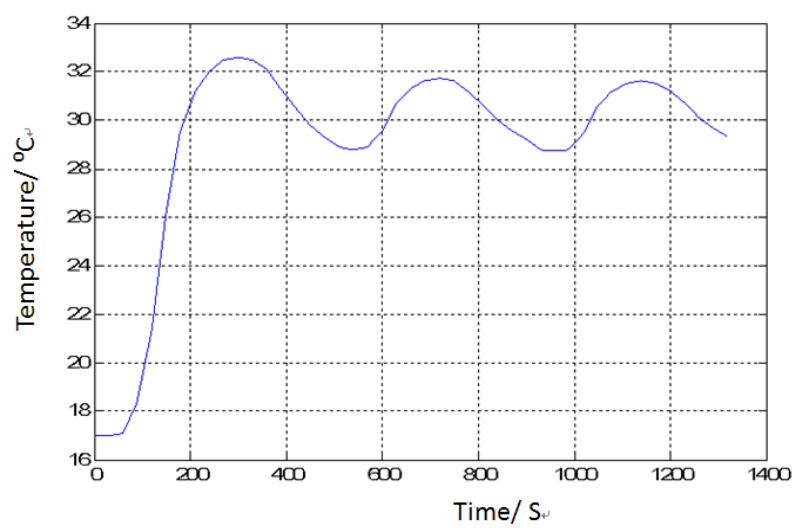

Fig.4. Effect diagram of PID control setting temperature $30^{\circ} \mathrm{C}$

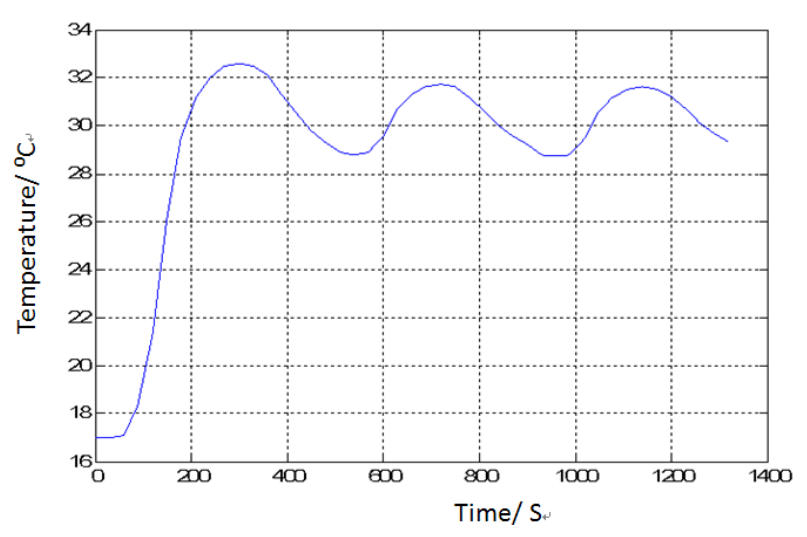

Fig.5. Effect diagram of fuzzy PID control setting temperature $30^{\circ} \mathrm{C}$

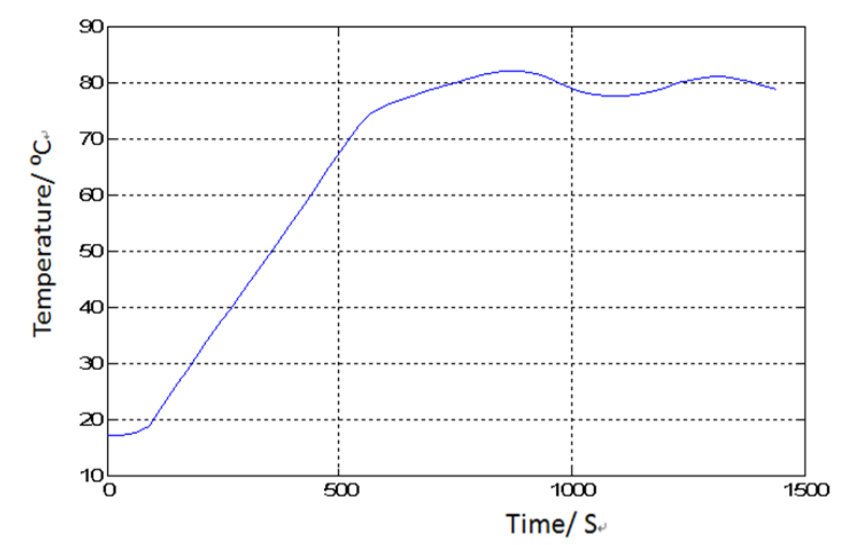

Fig.6. Effect diagram of PID control setting temperature $90^{\circ} \mathrm{C}$

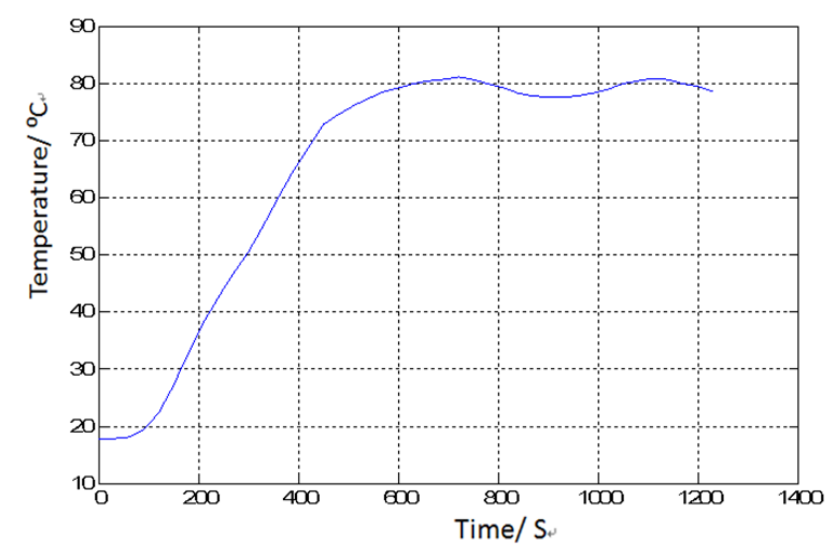

Fig.7. Effect diagram of fuzzy PID control setting temperature $90^{\circ} \mathrm{C}$

\section{CONCLUSION}

Through the simulation analysis, the fuzzy control with fuzzy self-tuning PID control compared with the conventional PID control, with better robustness and reliability. It has a very important role in the effective control of temperature in the process of agricultural production and bio-fermentation.

\section{REFERENCES}

[1] Ke Li, Temperature control system intelligent PID control algorithm, Wuhan: Huazhong University of Science and Technology, 2006.

[2] Kexing Wen, Research on Intelligent PID algorithm and its application in temperature control, Shanghai: Donghua University, 2009.

[3] Xiangquan Meng, PID parameter self-tuning method research and controller development, Dalian: Dalian University of Technology, 2010.

[4] Yimin Mao; Haifu Luo; Jing Zhang, Design and Simulation of a fuzzy self-tuning controller with PID parameters, automation and instrumentation, Vol. 1, pp.37-39, 2001.

[5] Zhen Liu; Xuezhi Jiang, Overview of PID controller parameter tuning method, Power System Automation, Vol. 21, pp.79-83, 1997.

[6] Zhenkuo Wu; Liang Zhang; Bo Zhang, PID parameter tuning and optimization. China High-tech Enterprise, Vol. 1, pp.16-17, 2010.

[7] K.S.Tang; Kim Fung Man; Guanrong Chen, An Optimal Fuzzy PID Controller, IEEE Transactions on industrial electronics, Vol. 1, pp.757$765,2007$. 